\title{
Factores socioeconómicos asociados \\ a retraso en talla en preescolares \\ asistentes a una institución educativa \\ de Floridablanca, Colombia
}

\author{
Socioeconomic factors associated \\ with stunting in preschool attending \\ an educational institution \\ of Floridablanca, Colombia
}

\begin{abstract}
Stunting is an indicator of human poverty and social development that causes high economic burden on countries. The objective of this study was to determine the association between socioeconomic factors and $z$ score height for age (HAZ) in preschool of the Colombo-German Foundation of Floridablanca, Colombia. A cross-sectional study was conducted. A random sample of 112 (2 to 5 years old) preschool of a institution for low income population was measured and weighed; their parents / caregivers answered a survey. The mean HAZ in preschool who live in households with more than one child (a) under 7 was lower compared with households with a child (a) under 7 years. Children living in housing with tenure called "possession untitled" scored lower mean HAZ compared with homeownership. Children living in households where the mother was the one who brings home were more HAZ compared with homes where the contributor was the father; this association remained in boys. In girls none of the variables of the final model was found associated with HAZ. It is important to strengthen programs aimed at improving the socioeconomic conditions of the mothers who are household heads.

Key words: Socioeconomic factors, child nutrition disorders, stunting, poverty, preschool.
\end{abstract}

\section{INTRODUCCIÓN}

La desnutrición crónica en menores de 5 años, evidenciada en el retraso de la talla, es un problema de salud asociado a factores socioeconómicos, acceso a servicios de saneamiento, alimentación familiar, nivel educativo y conocimientos de nutrición de los padres y desnutrición prenatal (1-4). Produce en el individuo un aumento de la morbimortalidad, afectando en la capacidad cognitiva y problemas de aprendizaje $(4,5)$. Está relacionado con un futuro caracterizado por menor productividad y remuneración económica (6) y ocasiona una carga económica y social a los países que la padecen (1).

La desnutrición crónica en el mundo ha venido disminuyendo entre el año 2000 y el 2013, de 199 millones (33\%) a
Adriana Angarita (1)

Gloria E. Prada $(1,2)$

Gloria L. Mendoza (1)

María N. Hernández (3)

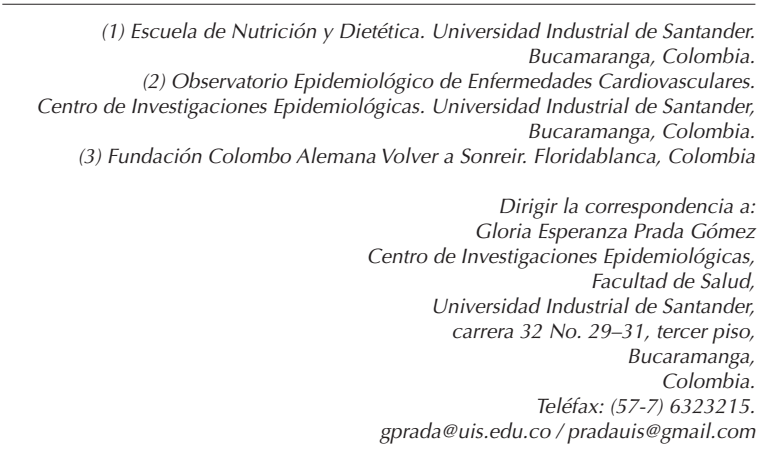

Este trabajo fue recibido el 16 de Octubre de 2015 aceptado con modificaciones el 29 de Diciembre de 2015 y aceptado para ser publicado el 14 de Marzo de 2016.

161 millones (25\%) respectivamente. El problema es alarmante en algunas regiones, en 2013 cerca de la mitad de todos los niños con desnutrición crónica vivían en Asia y un tercio en Africa (7). En América latina y el Caribe pasó de 13.8 millones en 1990 a 6.9 millones en 2012, equivalente al 12,8\% del total de los niños, Guatemala presenta la mayor prevalencia de la región con 48\%, seguido por Haití y Honduras con 30\%, Chile y Jamaica presentan la menor prevalencia, con un $2 \%$ y $5 \%$ respectivamente (8). En Colombia en 1990 el retraso en talla en menores de cinco años fue $26.1 \%$ y en el 2010 de $13.2 \%$ (9).

El retraso en talla en menores de cinco años es un indicador de pobreza y del nivel de desarrollo de una sociedad $(2,10,11)$, García y cols encontraron que en el quintil más 
pobre de la población Colombiana, la prevalencia de retraso en talla en menores de 5 años fue $21.8 \%$, mientras que en el quintil más rico de $4.5 \%$, además evidenciaron que vivir en zona urbana no es factor protector (2).

Más de 2.200 millones de personas en el mundo -15\% de la población- se encuentran en situación de pobreza multidimensional (12), este indicador tiene en cuenta cinco dimensiones, condiciones educativas, condiciones de la niñez y la juventud, características del trabajo, características del sector salud y servicios públicos y condiciones de la vivienda. En Colombia el año 2014 correspondió a 21.9\%, 15.4\% en las cabeceras municipales y $44.1 \%$ en los centros poblados y rural disperso (DANE). La pobreza extrema entendida la incapacidad para comprar la canasta básica de alimentos, entre 1990 y 2015 se redujo a la mitad, como lo plantean los objetivos del desarrollo del milenio, en Asia oriental, Asia sudoriental, América Latina y el Caribe y Cáucaso y Asia central, pero África subsahariana y Asia meridional no lo han logrado todavía (14). En Colombia fue $8.1 \%, 5.1 \%$ en cabeceras municipales y $18.0 \%$ en centros poblados y rural disperso (13).

El objetivo del estudio fue establecer los factores socioeconómicos asociados al retraso de talla en niños y niñas usuarios de la Fundación Colombo-Alemana y localizados en el municipio de Floridablanca, Santander, Colombia.

\section{SUJETOS Y MÉTODOS}

Estudio de corte transversal realizado durante los años 2013 y 2014. El universo estuvo constituido por 335 niños y niñas de dos a cinco años, que asisten de lunes a viernes de 7:00 a las 15:00 a la Fundación Colombo-Alemana. Una institución sin ánimo de lucro, creada para mejorar la calidad de vida y el fortalecimiento familiar de las comunidades en situación de vulnerabilidad del municipio de Floridablanca, el cual cuenta con una población estimada para el 2012 de 271,728 habitantes.

El presente estudio es un análisis secundario de una investigación que calculó un tamaño de muestra de 128 preescolares. Para este cálculo se consideró un nivel de confianza del $95 \%$, poder de $80 \%$, una precisión del 5,0\% y una prevalencia de exceso de peso esperada de $16,0 \%$ (15). Al tener en cuenta un $32,8 \%$ de pérdidas se seleccionaron de manera aleatoria 170 menores, de los cuales 112 representantes legales firmaron el consentimiento informado y aceptaron participar, siendo la tasa de participación de $66 \%$. La selección de la muestra se realizó mediante muestreo aleatorio simple a partir del listado de preescolares.

Previo a la recolección de la información, durante las reuniones de padres de familia se socializaron el proyecto y sus objetivos. Los padres o representantes de los niños y niñas seleccionados junto con la persona encargada de suministrar y preparar la alimentación al menor fueron citados en las instalaciones de la Fundación. Quienes no asistieron a la cita fueron abordados en reuniones regulares organizadas por la institución y en última instancia se realizaron visitas a los barrios o asentamientos donde vivían los menores.

Siete estudiantes del programa de Nutrición y Dietética de la Universidad Industrial de Santander y un nutricionista, entrenados en métodos de entrevista directa y mediciones antropométricas aplicaron la encuesta elaborada por el equipo de investigación (no validada) a madres (76,8\%), padres (7,1\%) y otros familiares $(16,1 \%)$. Se indagó por factores biológicos, económicos, sociodemográficos del niño(a) y sus familiares, antecedentes patológicos familiares de los padres, parentesco de la persona encargada del cuidado del niño(a). Se destaca que la estratificación socioeconómica colombiana, es una clasifica- ción de los domicilios o viviendas a partir de sus características físicas y de su entorno, en seis grupos o estratos: 1. Bajo-bajo, 2. Bajo, 3. Medio-bajo, 4. Medio, 5. Medio-alto, 6. Alto. Las personas víctimas del conflicto armando por desplazamiento fueron consideradas en estrato cero. La tasa de ocupación se calculó dividiendo el número de personas ocupadas sobre la población en edad de trabajar, constituida por todas las personas de 12 años en adelante. En los ingresos familiares se utilizó como referencia el salario mínimo legal vigente (SMLV) en Colombia para el año 2013, que correspondió a 589500 pesos colombianos (332 dólares USD).

Así mismo, los estudiantes de nutrición realizaron las mediciones de peso y talla a los preescolares. Para esto se siguieron las recomendaciones de la ISAK (International Standards for Anthropometrics Assessment) (16) y se utilizaron básculas digitales marca Tanita HD-314 con capacidad Máxima $150 \mathrm{~kg}$, con calibración automática $\mathrm{d}=0.1 \mathrm{~kg}$, tallímetros marca SECA Modelo 206. La prevalencia de retraso en talla para la edad se calculó teniendo en cuenta la clasificación de la Organización Mundial de la Salud. Así, después de obtener el Puntaje z de la talla para la edad (PZ-TE) utilizando el programa ANTHRO (17), los preescolares se clasificaron en: retraso en talla para la edad, si el PZ-TE estaba por debajo de -2 desviaciones estándar (DS), riesgo de retraso en talla para la edad si el PZ-TE entre $>=-2$ a $<-1$ DS y talla normal si el PZ-TE se encontraba $>=-1 \mathrm{DS}$, teniendo en cuenta lo establecido en la Resolución No. 2121 de 2010 del Ministerio de la Protección Social (18).

Las variables cualitativas se presentaron en frecuencias absolutas y relativas y sus respectivos intervalos de confianza del 95\%; en las variables cuantitativas se calculó la media aritmética y la desviación estándar o la mediana y el rango intercuartílico (percentil 25 - percentil 75) según la distribución de la variable evaluada con la prueba de Shapiro-Wilk. En el análisis bivariado se utilizaron modelos lineales generalizados (MLG) simples teniendo en cuenta el puntaje z del Índice de Masa Corporal (IMC) para la edad y sexo como variable dependiente. Con el fin de establecer si existían variables asociadas al puntaje $z$ del IMC para la edad y sexo, se aplicaron MLG múltiples. Se exploraron en el modelo final aquellas variables que obtuvieron en el bivariado un valor $p<0,20$, aunque se excluyeron las variables del padre: edad, escolaridad y ocupación, por tener 35 datos faltantes. El MLG es un modelo semiparamétrico para datos heterogéneos que no cumplen los supuestos de normalidad, adicionalmente por el método cuasi-likelihood no es necesario especificar la distribución de la variable respuesta; la interpretación de los coeficientes es directa y se logra el ajuste de sobredispersión de los datos (19). La evaluación del modelo final se realizó mediante la prueba linktest y se realizó el análisis de residuales deviance. Así mismo se aplicó el modelo a niños y niñas por separado (20). Los datos fueron digitados en el software EPIDATA 3.1 y exportados al software estadístico Stata 13.1 para su análisis (15).

Según la Resolución No. 008430 del 04 de octubre de 1993 del Ministerio de Salud de Colombia (21), esta investigación se consideró sin riesgo. Se garantizaron los principios éticos de respeto, autonomía, justicia y confidencialidad. El presente estudio fue aprobado por el Comité de Ética para la Investigación Científica de la Facultad de Salud de la Universidad Industrial de Santander y se solicitó el consentimiento informado por escrito del representante legal de los menores.

\section{RESULTADOS}

La muestra quedó conformada por 55 niñas y 57 niños, de los cuales $54,5 \%$ tenía entre cuatro y cinco años y $51,5 \%$ 
estaba en párvulos. (tabla 1). El 8,9\% ( $n=10)$ [IC95\% 4,8-15,9] presentaba con retraso en talla y $22,3 \%(n=25)$ IC95\% 15,5$31,1]$ con riesgo de retraso en talla. El promedio del PZ-TE fue $-0,64$ con un mínimo de $-3,38$ y un máximo 2,16. (tablas 1 y 2).

En relación a las características del hogar, se encontró una disminución significativa del PZ-TE en los preescolares que pertenecían a hogares con más de un niño menor de 7 años $(\beta=-0,43)$. En las demás variables no se encontró asociación significativa. (tabla 3).

En relación a las responsabilidades, niños cuidados por las madres presentaron un promedio mayor de PZ-TE comparado con aquellos cuidados por el padre $(\beta=0,80)$. Así mismo, también hubo un mayor promedio de PZ-TE cuando las abuelas o ambos padres decidían la alimentación el sábado $(\beta=0,032)$ comparado con la decisión realizada por la madre. (tabla 4).

En el análisis del modelo lineal generalizado múltiple, en toda la muestra continuó la asociación entre el número de menores de 7 años y el PZ-TE; así el PZ-TE en los preescolares que pertenecían a hogares con más de un niño(a) menor de 7 años fue menor comparado con los hogares con un niño (a) menor de 7 años. Los preescolares de hogares que vivían en una vivienda con tenencia denominada "posesión sin título" obtuvieron en promedio menor PZ-TE comparado con aquellos que vivían en vivienda propia, y los niños(as) de hogares donde la madre es quien aporta para el hogar tuvieron mayor PZ-TE comparado con los hogares en donde el que aporta es el padre; está misma asociación fue la única significativa para el subgrupo de niños varones, mientras que en el subgrupo de niñas ninguna de las variables del modelo final se encontró asociada a PZ-TE (tabla 5).

\section{DISCUSIÓN}

El retraso en talla es el resultado de múltiples causas, entre ellas, económicas, políticas, sociales y culturales $(3,4)$, sin embargo la pobreza es considerada como el principal determinante asociado a una alimentación insuficiente e inadecuada, a condiciones inadecuadas de saneamiento, la falta de acceso a servicios de salud y al aumento del riesgo de infecciones (8).

Tenencia de la vivienda. Los resultados del presente estudio, muestran una asociación negativa entre el tipo de tenencia "posesión sin título" y el retraso en talla. En Colombia para el año 2014, este tipo de tenencia correspondió a $2.8 \%$ los hogares, $1.8 \%$ en la cabecera municipal y $6.2 \%$ en los centros poblados y rural disperso (22). Estos asentamientos surgen como una opción para aquellos hogares que no cuentan con recursos para comprar vivienda o cancelar un arriendo. Su ubicación en la mayoría de los casos se realiza en lugares de alto riesgo y las viviendas son construidas con cartón, lata, madera, teja entre otros (23). Las familias que allí residen, tienen limitados ingresos económicos, falta de trabajo y un acceso disminuido y prolongado de alimentos (24-27), falta de agua potable, inadecuadas condiciones ambientales $(1-4,8)$, todo lo anterior genera afectación del estado nutricional y de la salud de la población y en el caso de los niños un retraso de la talla, situación que se evidencia en esta investigación.

Madre cabeza de hogar, única responsable de la familia. Existe una asociación entre el ingreso familiar con el estado nutricional de los hijos y el acceso a los alimentos. Un estudio encontró que el IMC de los niños es mayor cuando los dos padres trabajaban (28), otra investigación mostró que la cantidad de alimentos que se compra en las familias esta determinada por el número de miembros que trabajaban y aportan al sostenimiento familiar (25). En los hogares donde la madre es la única responsable del sustento familiar, la cantidad y calidad de alimentos disponibles se ve disminuida, sobre todo si ella no tiene un ingreso estable o si la remuneración que recibe por su actividad laboral es poca, situación que repercute en el estado nutricional de la familia, especialmente de los hijos preescolares. Otro aspecto a considerar es la calidad del cuidado que reciben los niños, en familias vulnerables los compromisos laborales de las madres obligan a dejar esta responsabilidad a cargo de otras personas, que en muchos casos no están preparados ni comprometidos con esta función, varios estudios han mostrado que cuando el cuidado es ofrecido por personas distintas a los padres se incrementa la malnutrición del niño $(2,17,29,30)$.

En Colombia se encontró mayor prevalencia de desnutrición crónica (30,5\%) en los hijos de madres cabezas de hogar, comparados con hogares en que están los dos padres $(24,7 \%)$, aunque no se asoció estadísticamente (30). En Colombia en el año 2014, en $34.7 \%$ de los hogares fue la madre la única responsable del sostenimiento de la familia (22), en este estudio correspondió a $29.5 \%$ y se asoció al retraso en talla.

Hogares con más de un niño menor de 7 años. Un mayor número de niños menores de 7 años en la familia, exige mayores recursos económicos y tiempo para cuidarlos. Un estudio realizado en el Perú mostró que tener 2 o más hijos

TABLA 1

Características de los niños y niñas de la Fundación Colombo-Alemana asociadas al puntaje z talla para la edad (PZ-TE). Floridablanca, Colombia, 2013.

Variable

Femenino

Masculino

Edad

2-3 años

4-5 años
Medida de resumen

$\%$

IC95\%
Puntaje z del IMC para edad y sexo

Mediana P25 P75

$\mathrm{IMC}=$ Indice de masa corporal. $\mathrm{P} 25=$ Percentil $25 . \mathrm{P} 75=$ Percentil $75 . \mathrm{n}=$ frecuencia absoluta.

$\%=$ Porcentaje. IC95\% Intervalo de confianza del 95\%. Ref.=Grupo de referencia. 
en el hogar se asoció a desnutrición crónica $(10,31)$, otros estudios en Colombia evidenciaron asociación con el número de hermanos o de integrantes de la familia $(32,33)$. El tamaño familiar determina la disponibilidad, calidad y consumo de alimentos al interior de la familia, de otra parte cuando existe un mayor número de preescolares, se requiere más tiempo para satisfacer las necesidades de cuidado, los estudios muestran asociación entre los cuidados que el niño recibe y su estado nutricional $(2,17,29)$.

Limitaciones del estudio. Es reconocido que el entorno de la madre durante el embarazo incide en el desarrollo intrauterino y que en condiciones de restricción, el bajo peso al nacer se presenta y es una causa del retraso del crecimiento pos nacimiento (2), este estudio no evaluó el peso al nacer

TABLA 2

Características de las madres y los padres de los niños y niñas de la Fundación Colombo-Alemana asociadas al puntaje z talla para la edad (PZ-TE). Floridablanca, Colombia, 2013.

\begin{tabular}{|c|c|c|c|c|c|c|c|c|c|c|}
\hline \multirow[t]{2}{*}{ Variable } & \multirow{2}{*}{\multicolumn{2}{|c|}{$\begin{array}{l}\text { Medida de } \\
\text { resumen }{ }^{1}\end{array}$}} & \multicolumn{2}{|c|}{ IC95\% ${ }^{2}$} & \multicolumn{2}{|c|}{ PZ-TE } & \multicolumn{4}{|c|}{ Modelo lineal simple } \\
\hline & & & & & Media & $\mathrm{DE}$ & $\begin{array}{c}\text { Coeficiente } \\
\text { Beta }\end{array}$ & & $5 \%$ & Valor $\mathrm{p}$ \\
\hline \multicolumn{11}{|l|}{ Características de la madre } \\
\hline Edad (años) $)^{3}$ & 27 & $23-33$ & 25,0 & 28,0 & - & - & 0,01 & $-0,02$ & 0,04 & 0,558 \\
\hline \multicolumn{11}{|l|}{ Estado civil } \\
\hline $\begin{array}{l}\text { Union libre/casada } \\
\text { Separada/Divorciada }\end{array}$ & 79 & 70,5 & 62,1 & 79 & $-0,70$ & 1,01 & Ref. & & & \\
\hline /viuda/Madre Soltera & 33 & 29,5 & 21,0 & 37,9 & $-0,51$ & 0,91 & 0,19 & $-0,21$ & 0,59 & 0,346 \\
\hline \multicolumn{11}{|l|}{ Escolaridad } \\
\hline $\begin{array}{l}\text { Ninguno- } \\
\text { BasicaPrimaria } \\
\text { Secundaria }\end{array}$ & 28 & 26,7 & 17 & 33 & $-0,47$ & 1,08 & Ref. & & & \\
\hline Tecnico/Tecnologo & 77 & 73,3 & 60,2 & 77,3 & $-0,71$ & 0,96 & $-0,24$ & $-0,67$ & 0,20 & 0,281 \\
\hline \multicolumn{11}{|l|}{ Ocupación } \\
\hline Trabajando & 70 & 62,5 & 53,5 & 71,5 & $-0,56$ & 0,94 & Ref. & & & \\
\hline \multicolumn{8}{|l|}{ Buscando trabajo/ } & \multicolumn{2}{|c|}{ Estudiando/Oficios } & \\
\hline del hogar & 42 & 37,5 & 28,5 & 46,5 & $-0,78$ & 1,03 & $-0,22$ & $-0,59$ & 0,16 & 0,260 \\
\hline \multicolumn{11}{|l|}{ Características del padre } \\
\hline \multicolumn{11}{|l|}{ Padre vive en el hogar } \\
\hline $\mathrm{Si}$ & 79 & 70,5 & 62,1 & 79 & $-0,69$ & 1,00 & Ref. & & & \\
\hline No & 33 & 29,5 & 21 & 37,9 & $-0,53$ & 0,93 & $-0,02$ & 0,16 & $-0,24$ & 0,570 \\
\hline Edad (años)4 & 32 & $28-37$ & 30,0 & 34,0 & - & - & $-0,01$ & $-0,02$ & 0,00 & 0,087 \\
\hline \multicolumn{11}{|l|}{ Estado civil } \\
\hline Union libre/Casado & 76 & 69,7 & 59,2 & 76,5 & $-0,68$ & 1,02 & Ref. & & & \\
\hline $\begin{array}{l}\text { Separado/Divorciado/ } \\
\text { viudo/Padre Soltero }\end{array}$ & 33 & 30,3 & 21,0 & 37,9 & $-0,68$ & 0,77 & 0,00 & $-0,39$ & 0,39 & 0,999 \\
\hline \multicolumn{11}{|l|}{ Escolaridad } \\
\hline Ninguno-Primaria & 34 & 30,4 & 21,8 & 38,9 & $-0,76$ & 1,21 & Ref. & & & \\
\hline \multicolumn{10}{|l|}{ Tecnico/Tecnologo/ } & 0,650 \\
\hline Universitario & 5 & 4,5 & 0,6 & 8,3 & $-0,66$ & 0,73 & 0,27 & $-0,67$ & 1,20 & 0,573 \\
\hline Sin dato & 34 & 30,4 & 21,8 & 38,9 & $-0,49$ & 1,49 & 0,23 & $-0,24$ & 0,71 & 0,334 \\
\hline \multicolumn{11}{|l|}{ Ocupación } \\
\hline Trabajando & 74 & 66,1 & 57,3 & 74,8 & $-0,70$ & 1,03 & Ref. & & & \\
\hline \multicolumn{11}{|l|}{ Buscando trabajo/ } \\
\hline Estudiando & 3 & 2,7 & 0,0 & 5,7 & $-0,47$ & 0,58 & 0,23 & $-0,92$ & 1,38 & 0,688 \\
\hline Sin registro & 35 & 31,2 & 22,7 & 39,8 & $-0,53$ & 0,91 & 0,17 & $-0,23$ & 0,57 & 0,410 \\
\hline
\end{tabular}


debido a la imposibilidad de contar con el documento oficial en el cual se registra esta información.

\section{CONCLUSIÓN}

El retraso en talla en menores de cinco años se asoció de manera inversa con el tipo de tenencia "posesión sin título", la responsabilidad única de la madre con el sustento del hogar y el número de menores de 7 años. Aunque los niños participantes tenían garantizada la atención en la institución educativa de lunes a viernes hasta las 16:00 horas, y la satisfacción del $70 \%$ de requerimientos nutricionales mediante la alimentación ofrecida, la pregunta es ¿cuál será la situación de

TABLA 3

Características socioeconómicas de los hogares de los niños y niñas de la Fundación Colombo-Alemana asociadas al puntaje z talla para la edad (PZ-TE).Floridablanca, Colombia, 2013.

\begin{tabular}{|c|c|c|c|c|c|c|c|c|c|c|}
\hline \multirow{3}{*}{$\begin{array}{l}\text { Variable } \\
\text { Tasa de ocupación }\end{array}$} & \multirow{2}{*}{\multicolumn{2}{|c|}{$\begin{array}{l}\text { Medida de } \\
\text { resumen }{ }^{1}\end{array}$}} & \multirow{2}{*}{\multicolumn{2}{|c|}{ IC95\%² }} & \multicolumn{2}{|c|}{ PZ-TE } & \multicolumn{4}{|c|}{ Modelo lineal simple } \\
\hline & & & & & \multirow[t]{2}{*}{ Media } & \multirow[t]{2}{*}{$\mathrm{DE}$} & \multirow{2}{*}{$\begin{array}{l}\text { Coeficiente } \\
\qquad-0,01\end{array}$} & \multicolumn{2}{|c|}{$\mathrm{IC95 \%}$} & \multirow{2}{*}{$\begin{array}{l}\text { Valor } \mathrm{p} \\
0,981\end{array}$} \\
\hline & 0,66 & 0,26 & 0,61 & 0,71 & & & & $-0,72$ & 0,70 & \\
\hline \multicolumn{11}{|c|}{ Estrato socioeconomico } \\
\hline Estrato cero & 32 & 28,6 & 20,2 & 36,9 & $-0,81$ & 0,88 & Ref. & & & \\
\hline Estrato 1 y 2 & 80 & 71,4 & 63,1 & 79,8 & $-0,57$ & 1,01 & 0,24 & $-0,17$ & 0,64 & 0,252 \\
\hline \multicolumn{11}{|l|}{ Ingresos familiares } \\
\hline 0-0,5 SMLV & 14 & 12,5 & 6,4 & 18,6 & $-0,87$ & 0,65 & Ref. & & & \\
\hline $0,6-1,0$ SMLV & 38 & 33,9 & 25,2 & 42,7 & $-0,64$ & 1,15 & 0,23 & $-0,37$ & 0,84 & 0,444 \\
\hline 1,1-1,5 SMLV & 28 & 25,0 & 17,0 & 33,0 & $-0,38$ & 0,95 & 0,50 & $-0,14$ & 1,13 & 0,123 \\
\hline 1,5-3,9 SMLV & 32 & 28,6 & 20,2 & 36,9 & $-0,78$ & 0,88 & 0,09 & $-0,53$ & 0,71 & 0,763 \\
\hline \multicolumn{11}{|l|}{ Procedencia n \% } \\
\hline Urbana & 74 & 69,8 & 57,3 & 74,8 & $-0,57$ & 0,95 & Ref. & & & \\
\hline Rural & 32 & 30,2 & 20,2 & 36,9 & $-0,79$ & 1,08 & $-0,22$ & $-0,64$ & 0,19 & 0,288 \\
\hline \multicolumn{11}{|l|}{ Familia es desplazada } \\
\hline No & 62 & 57,4 & 46,2 & 64,6 & $-0,63$ & 1,01 & Ref. & & & \\
\hline $\mathrm{Si}$ & 46 & 42,6 & 32,0 & 50,2 & $-0,65$ & 0,96 & $-0,01$ & $-0,40$ & 0,37 & 0,942 \\
\hline \multicolumn{11}{|c|}{ Recibe subsidios gubernamentales de ONG o amigos para el sostenimiento } \\
\hline No & 41 & 36,6 & 27,7 & 45,5 & $-0,72$ & 0,93 & Ref. & & & \\
\hline $\mathrm{Si}$ & 71 & 63,4 & 54,5 & 72,3 & $-0,60$ & 1,01 & 0,12 & $-0,26$ & 0,50 & 0,529 \\
\hline \multicolumn{11}{|l|}{ Tenencia de vivienda } \\
\hline Propia & 36 & 32,1 & 23,5 & 40,8 & $-0,47$ & 0,90 & Ref. & & & \\
\hline Arrendada & 47 & 42,0 & 32,8 & 51,1 & $-0,61$ & 1,11 & $-0,14$ & $-0,57$ & 0,28 & 0,508 \\
\hline Posesión sin título & 29 & 25,9 & 17,8 & 34,0 & $-0,90$ & 0,81 & $-0,43$ & $-0,91$ & 0,05 & 0,080 \\
\hline \multicolumn{11}{|c|}{ Número de personas que conforman el núcleo familiar incluido el niño } \\
\hline $1-4$ & 58 & 54,2 & 42,5 & 61,0 & $-0,47$ & 1,04 & Ref. & & & \\
\hline $5-9$ & 49 & 45,8 & 34,6 & 52,9 & $-0,78$ & 0,87 & $-0,31$ & $-0,68$ & 0,07 & 0,105 \\
\hline \multicolumn{11}{|c|}{ Número de personas que vive con el niño } \\
\hline $1-3$ & 52 & 46,4 & 37,2 & 55,7 & $-0,52$ & 1,06 & Ref. & & & \\
\hline $4-9$ & 60 & 53,6 & 44,3 & 62,8 & $-0,75$ & 0,89 & $-0,22$ & $-0,59$ & 0,14 & 0,231 \\
\hline \multicolumn{11}{|c|}{ Número de personas que duermen con el niño en el mismo cuarto } \\
\hline $0-1$ & 60 & 53,6 & 44,3 & 62,8 & $-0,69$ & 0,94 & Ref. & & & \\
\hline $2-4$ & 52 & 46,4 & 37,2 & 55,7 & $-0,58$ & 1,03 & 0,11 & $-0,26$ & 0,48 & 0,557 \\
\hline \multicolumn{11}{|c|}{ Número de niños menores de 7 años en el hogar } \\
\hline 1 & 60 & 53,6 & 44,3 & 62,8 & $-0,44$ & 0,95 & Ref. & & & \\
\hline $2-4$ & 52 & 46,4 & 37,2 & 55,7 & $-0,87$ & 0,97 & $-0,43$ & $-0,79$ & $-0,07$ & 0,021 \\
\hline \multicolumn{11}{|l|}{ Tipo de familia } \\
\hline Extensa & 40 & 35,7 & 26,8 & 44,6 & $-0,51$ & 1,01 & Ref. & & & \\
\hline Nuclear & 65 & 58,0 & 48,9 & 67,2 & $-0,73$ & 1,01 & $-0,22$ & $-0,61$ & 0,17 & 0,261 \\
\hline Monoparental & 7 & 6,2 & 1,8 & 10,7 & $-0,53$ & 0,30 & $-0,02$ & $-0,82$ & 0,77 & 0,954 \\
\hline $\begin{array}{l}I M C=\text { Indice de masa corpo } \\
\text { SMVL= Salarios mínimos le } \\
\text { 1 Promedio y desviación est }\end{array}$ & variab & a de o & Frecu & bsolut & fianza d & n la & ariables. & & & \\
\hline
\end{tabular}




\section{TABLA 4}

Responsables del cuidado, alimentación, educación y sostenimiento económico de los hogares de los niños y niñas de la Fundación Colombo-Alemana asociadas al puntaje z talla para la edad (PZ-TE). Floridablanca, Colombia, 2013.

\begin{tabular}{|c|c|c|c|c|c|c|c|c|c|c|}
\hline \multirow{3}{*}{$\begin{array}{l}\text { Persona que... } \\
\text { Cuida al niño }\end{array}$} & \multicolumn{3}{|c|}{ Medida de resumen } & & \multicolumn{2}{|c|}{ PZTE } & \multicolumn{4}{|c|}{ Modelo lineal simple } \\
\hline & \multirow[t]{2}{*}{$\mathrm{n}$} & \multirow[t]{2}{*}{$\%$} & \multicolumn{2}{|c|}{ IC95\% } & \multirow{2}{*}{ Media } & \multirow[t]{2}{*}{$\mathrm{DE}$} & \multirow[t]{2}{*}{ Coeficiente } & \multicolumn{2}{|c|}{ IC95\% } & \multirow[t]{2}{*}{ Valor $\mathrm{p}$} \\
\hline & & & & & & & & & & \\
\hline Madre & 82 & 73,9 & 65,0 & 81,4 & $-0,70$ & 0,99 & Ref. & & & \\
\hline Abuela(o) & 20 & 18,0 & 10,8 & 25,0 & $-0,61$ & 1,03 & 0,09 & $-0,39$ & 0,58 & 0,710 \\
\hline Otro familiar & 9 & 8,1 & 3,0 & 13,1 & $-0,20$ & 0,75 & 0,50 & $-0,18$ & 1,19 & 0,149 \\
\hline \multicolumn{11}{|c|}{ Aporta para el sustento del hogar } \\
\hline Padre & 41 & 36,6 & 27,7 & 45,5 & $-0,68$ & 1,07 & Ref. & & & \\
\hline Madre & 14 & 12,5 & 6,4 & 18,6 & 0,12 & 0,94 & 0,80 & 0,21 & 1,38 & 0,008 \\
\hline Padre y Madre & 34 & 30,4 & 21,8 & 38,9 & $-0,87$ & 0,92 & $-0,19$ & $-0,62$ & 0,25 & 0,394 \\
\hline Otros & 12 & 10,7 & 5,0 & 16,4 & $-0,62$ & 0,83 & 0,06 & $-0,56$ & 0,68 & 0,850 \\
\hline \multicolumn{11}{|l|}{ Padre y/o Madre } \\
\hline y otros & 11 & 9,8 & 4,3 & 15,3 & $-0,78$ & 0,61 & $-0,10$ & $-0,74$ & 0,54 & 0,756 \\
\hline \multicolumn{11}{|c|}{ Responde por la preparación de alimentos de lunes a viernes } \\
\hline Madre & 86 & 76,8 & 69,0 & 84,6 & $-0,68$ & 0,99 & Ref. & & & \\
\hline Abuela & 19 & 17,0 & 10,0 & 23,9 & $-0,56$ & 1,00 & 0,13 & $-0,37$ & 0,62 & 0,611 \\
\hline Otro & 7 & 6,2 & 1,8 & 10,7 & $-0,38$ & 0,90 & 0,30 & $-0,46$ & 1,07 & 0,436 \\
\hline \multicolumn{11}{|c|}{ Responde por la preparación de alimentos el sábado } \\
\hline Madre & 80 & 71,4 & 63,1 & 79,8 & $-0,69$ & 0,96 & Ref. & & & \\
\hline Abuela & 17 & 15,2 & 10,8 & 25,0 & $-0,40$ & 1,14 & 0,30 & $-0,22$ & 0,82 & 0,263 \\
\hline Otro & 15 & 13,4 & 7,8 & 20,8 & $-0,65$ & 0,93 & 0,05 & $-0,50$ & 0,59 & 0,869 \\
\hline \multicolumn{11}{|c|}{ Responde por la preparación de alimentos el domingo } \\
\hline Madre & 76 & 67,9 & 59,2 & 76,5 & $-0,66$ & 0,95 & Ref. & & & \\
\hline Abuela & 20 & 17,9 & 8,5 & 21,8 & $-0,52$ & 1,19 & 0,13 & $-0,36$ & 0,62 & 0,591 \\
\hline Otro & 16 & 14,3 & 7,1 & 19,7 & $-0,72$ & 0,89 & $-0,07$ & $-0,60$ & 0,47 & 0,810 \\
\hline \multicolumn{11}{|c|}{ Decide alimentación de lunes a viernes } \\
\hline $\begin{array}{l}\text { Madre } \\
\text { Abuela/ }\end{array}$ & 78 & 69,6 & 61,1 & 78,2 & $-0,64$ & 0,99 & Ref. & & & \\
\hline Ambos padres & 22 & 19,6 & 12,3 & 27,0 & $-0,71$ & 1,03 & 0,02 & $-0,46$ & 0,49 & 0,939 \\
\hline Otro familiar/ & & & & & & & & & & \\
\hline Vecina-amiga & 6 & 5,4 & 1,2 & 9,5 & $-0,84$ & 1,48 & $-0,20$ & $-1,03$ & 0,63 & 0,634 \\
\hline Niño & 6 & 5,4 & 1,2 & 9,5 & $-0,36$ & 0,67 & 0,03 & $-0,80$ & 0,86 & 0,944 \\
\hline Decide alimentació & el sá & & & & & & & & & \\
\hline $\begin{array}{l}\text { Madre } \\
\text { Abuela/ }\end{array}$ & 72 & 64,3 & 55,4 & 73,2 & $-0,73$ & 0,92 & Ref. & & & \\
\hline $\begin{array}{l}\text { Ambos padres } \\
\text { Otro familiar/ }\end{array}$ & 23 & 20,5 & 16,2 & 32,0 & $-0,30$ & 1,13 & 0,50 & 0,04 & 0,96 & 0,032 \\
\hline Vecina-amiga & 13 & 11,6 & 4,3 & 15,3 & $-0,89$ & 1,14 & $-0,15$ & $-0,73$ & 0,42 & 0,597 \\
\hline Niño & 4 & 3,6 & 0,6 & 8,3 & 0,02 & 0,50 & 0,14 & $-0,84$ & 1,13 & 0,775 \\
\hline Decide alimentació & el d & & & & & & & & & \\
\hline $\begin{array}{l}\text { Madre } \\
\text { Abuela/ }\end{array}$ & 69 & 61,6 & 52,6 & 70,6 & $-0,73$ & 0,96 & Ref. & & & \\
\hline $\begin{array}{l}\text { Ambos padres } \\
\text { Otro familiar/ }\end{array}$ & 27 & 24,1 & 13,1 & 28,0 & $-0,36$ & 1,16 & 0,40 & $-0,03$ & 0,84 & 0,070 \\
\hline Vecina-amiga & 11 & 9,8 & 5,7 & 17,5 & $-0,82$ & 1,08 & $-0,09$ & $-0,71$ & 0,54 & 0,786 \\
\hline Niño & 5 & 4,5 & 0,1 & 7,0 & $-0,24$ & 0,62 & 0,06 & $-0,84$ & 0,95 & 0,899 \\
\hline Decide respecto a I & educ & n de los & & & & & & & & \\
\hline Padre & 5 & 4,5 & 0,6 & 8,3 & $-0,64$ & 0,82 & Ref. & & & \\
\hline Madre & 37 & 33,0 & 24,3 & 41,7 & $-0,60$ & 1,04 & 0,04 & $-0,90$ & 0,98 & 0,933 \\
\hline Padre y Madre & 65 & 58,0 & 48,9 & 67,2 & $-0,68$ & 0,98 & $-0,04$ & $-0,95$ & 0,87 & 0,931 \\
\hline Otros & 5 & 4,5 & 0,6 & 8,3 & $-0,46$ & 0,79 & 0,18 & $-1,06$ & 1,43 & 0,770 \\
\hline Acompaña al niño & ientr & nsume & nentos & & & & & & & \\
\hline Ambos padres & 46 & 41,1 & 32,0 & 50,2 & $-0,63$ & 0,67 & Ref. & & & \\
\hline Madre & 39 & 34,8 & 26,0 & 43,6 & $-0,72$ & 1,27 & $-0,09$ & $-0,52$ & 0,33 & 0,672 \\
\hline Varios familiares & 20 & 17,9 & 10,8 & 25,0 & $-0,65$ & 0,97 & $-0,02$ & $-0,55$ & 0,50 & 0,926 \\
\hline Solo & 7 & 6,2 & 1,8 & 10,7 & $-0,29$ & 0,95 & 0,34 & $-0,46$ & 1,13 & 0,400 \\
\hline
\end{tabular}


aquellos preescolares que no acceden a este tipo de programas de bienestar en la primera infancia?.

Los resultados de este estudio evidencian la necesidad de reforzar las políticas de los objetivos del milenio de erradicar el hambre, las políticas de primera infancia y la importancia de fortalecer los programas que buscan el mejoramiento de las condiciones socioeconómicas de las madres cabeza de hogar.

\section{RESUMEN}

El retraso en talla es un indicador de pobreza y desarrollo social que ocasiona elevada carga económica a los países. El objetivo del presente estudio fue determinar la asociación entre factores socioeconómicos y puntaje z talla para la edad (PZ-TE) en preescolares de la Fundación Colombo-Alemana de Floridablanca, Colombia. Se realizó un estudio de corte transversal. Una muestra aleatoria de 112 (2-5 años) preescolares de una institución que tiende población de bajos recursos fue medida y pesada; sus padres/cuidadores respondieron una encuesta. El promedio del PZ-TE en preescolares de hogares con más de un niño(a) menor de 7 años fue menor comparado con los hogares con un niño(a) menor de 7 años. Quienes vivían en viviendas con tenencia denominada "posesión sin título" obtuvieron en promedio menor PZ-TE comparado con la vivienda propia. Los niños(as) de hogares donde la madre es quien aporta para el hogar tuvieron mayor PZ-TE comparado con los hogares en donde el que aporta es el padre; está asociación se mantuvo en niños varones. En las niñas ninguna de las variables del modelo final se encontró asociada a PZ-TE. Es importante fortalecer los programas que buscan el mejoramiento de las condiciones socioeconómicas de las madres cabeza de hogar.

Palabras clave. Factores socioeconómicos, desnutrición, retraso en talla, pobreza, preescolar.

\section{TABLA 5}

Factores asociados al puntaje z talla para la edad (PZ-TE) en los niños y niñas de la Fundación Colombo-Alemana de Floridablanca, Colombia (2013). Análisis Multivariado usando Modelo lineal múltiple.

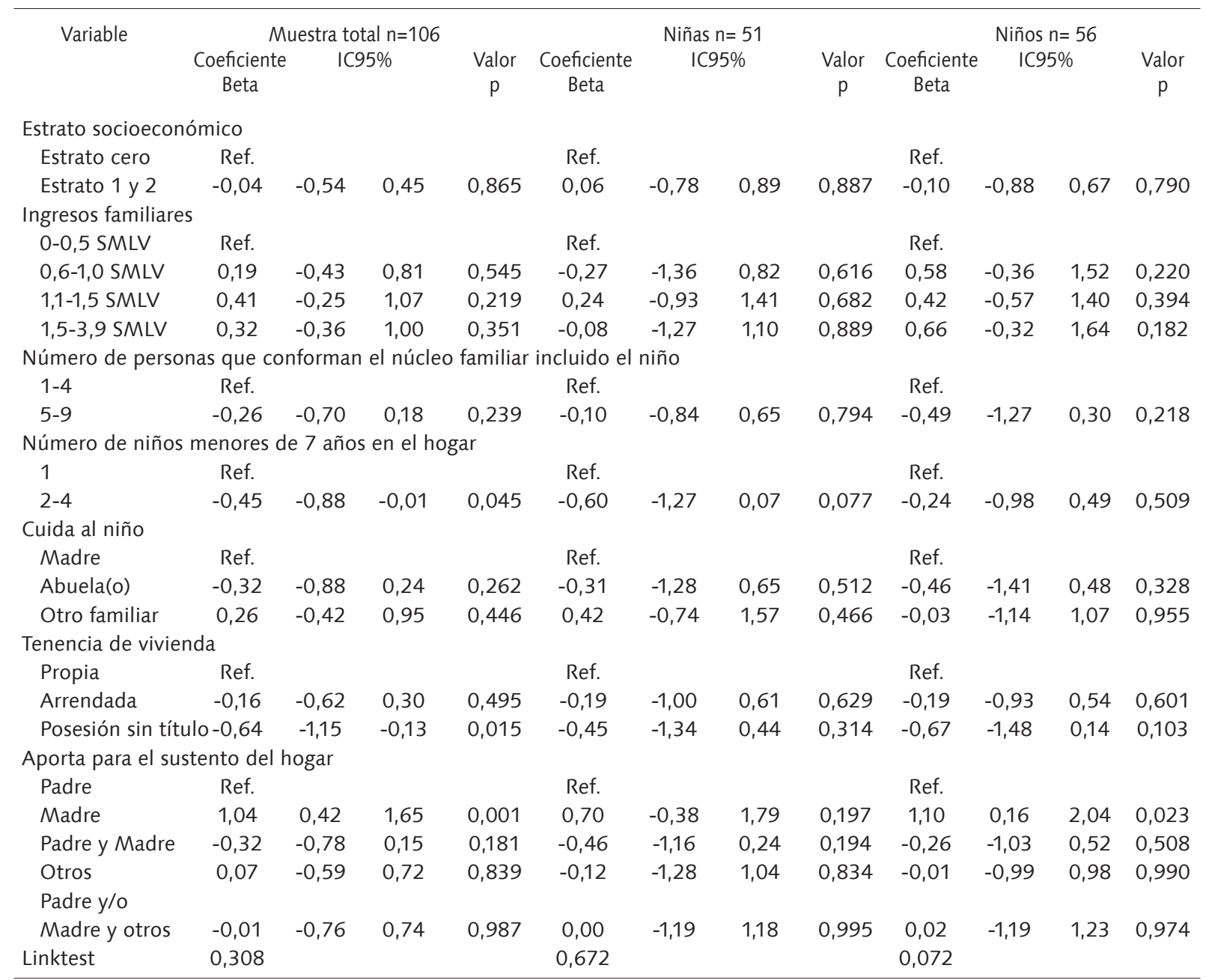

IC95\% = Intervalo de confianza del 95\%. SMLV= Salario mínimo legal vigente. - = No aplica. Ref.= Grupo de referencia. 


\section{BIBLIOGRAFÍA}

1. Jesmin A, Yamamoto SS, Malik AA, Haque MA. Prevalence and Determinants of Chronic Malnutrition among Preschool Children: A Cross-sectional Study in Dhaka City, Bangladesh. J Health Popul Nutr. 2011;29(5):494-9.

2. Garcia S, Sarmiento OL, Forde I, Velasco T. Socio-economic inequalities in malnutrition among children and adolescents in Colombia: the role of individual-, household- and community-level characteristics. Public Health Nutr. 2013;16(9):1703-18.

3. de Onis M1, Blössner M, Borghi E. Prevalence and trends of stunting among pre-school children, 1990-2020. Public Health Nutr. 2012;15(1):142-8.

4. Waterlow JC. Introduction. Causes and mechanisms of linear growth retardation (stunting). Eur J Clin Nutr. 1994;48 (Supp/ 1):S1-4.

5. Martorell $R$. The nature of child malnutrition and its longterm implications. Food Nutr Bull. 1999;20(3):288-92.

6. Victora CG, Adair L, Fall C, Hallal PC, Martorell $R$, Richter $L$, et al. Maternal and child undernourishment: consequences for adult health and human capital. Lancet. 2008;371(9609):340-57.

7. UNICEF, WHO, WORLD BANK. Levels and trends in child malnutrition 2014. Available in http://www.who.int/nutgrowthdb/summary_jme_2013.pdf?ua=1\#page=1\&zoom= auto,-107,848. Retrieved June 2015.

8. FAO. Food and Agriculture Organization in Latin America and the Caribbean. 2013. FAO, 2014. Available in http:// www.fao.org/docrep/019/i3520s/i3520s.pdf. Retrieved June 2015.

9. Instituto Colombiano de Bienestar Familiar. National survey of the Nutritional State in Colombia.-ENSIN 2010 -. Bogota. 2011.

10. Sobrino M, Gutiérrez C, Cunha AJ, Dávila M, Alarcón J. Malnutrition in under-five-year-old children in Peru: trends and determining causes. Rev Panam Salud Pública. 2014;35(2):104-12.

11. Menon P, Ruel MT, Morris SS. Socioeconomic Differentials in Child Stunting are Consistently Larger in Urban than in Rural Areas. Food and Nutrition Discussion Paper no. 97. Washington, DC: Internat Food Policy Res Inst; 2000.

12. PUND Program of The United Nations 3017917948 for the development. Summary: Human development report 2014. [Internet] New York 2014. Available in http://www.undp. org/content/dam/undp/library/corporate/HDR/2014HDR/ HDR-2014-Summary-Spanish.pdf. Retrieved April 2015.

13. DANE, Colombia. Monetary poverty and Multidimensional. Main results 2014. Available in http://www.dane.gov.co/ files/investigaciones/condiciones_vida/pobreza/. Retrieved April 2015.

14. UN. Millennium goals report 2014. Available in http://www. un.org/es/millenniumgoals/pdf/mdg-report-2014-spanish. pdf. Retrieved April 2015.

15. StataCorp. Stata Statistical Software: Release 13. Texas, United States of America: StataCorp LP; 2013.

16. Stewart A, Marfell-Jones M, Olds T, de Ridder H. International standards for anthropometric assessment. Lower Hutt, New Zealand: ISAK; 2011.

17. World Health Organization. Software ANTHRO 3.1. Geneva, Switzerland.: WHO; 2010.

18. Health and Social Protection Ministry. Colombia. Resolution 002121 June 9th 2010. In which the growth patterns posted by the WHO in 2006 and 2007 are taken on infants and and adolescents between 0 and 18 years old. Available in http://www.minsalud.gov.co/sites/rid/Lists/BibliotecaDigital. Retrieved June 2015.

19. Fonseca M de J, Andreozzi VL, Faerstein E, Chor D, Carvalho MS. Alternatives in modeling of body mass index as a continuous response variable and relevance of residual analysis. Cad Saude Publica. 2008;24(2):473-8.

20. Lauritsen JM, Bruus M, Myatt MA. An extended tool for validated dataentry and documentation of data. Odense, Denmark: The EpiData Association; 2001.

21. Health and Social Protection Ministry. Colombia. Resolution 008430 Octubre 4th 1993. Based on it, the scientific, technical and administrative regulations are stablished for former investigations in health. 008430 (1993). Available in http://www.invima.gov.co/images/pdf/medicamentos/ resoluciones. Retrieved May 2015.

22. DANE, Colombia. National survey of Quality of Life - ENCV 2013-. Reported on May 26 2014. Available in http:// www.dane.gov.co/index.php/esp/estadisticas-sociales/ calidad-de-vida-ec. Retrieved June 2015.

23. Wiesenfeld E. Between the invasion and consolidation of neighborhoods: Analysis of the resistance against eviction. Estud Psicol. 1998; 3(1): 33-51.

24. PMA, European Commission, PAHO. FAO. Nutritional state of food and health conditions of the internally displaced population by the violence in six subregions of the country. Final report. Bogota; 2005.

25. Prada GE, Herrán OF, Ortiz R. Food pattern and access to diet of the internally displaced families in the town of Girón, Santander, Colombia. Rev Panam Public Health 2008; 23(4):257-63.

26. Gamboa EM, López N, Vera LM, Prada GE. Food pattern and nutritional state in internally displaced children in Piedecuesta, Colombia. Rev Public Health 2007; 9(1):129-39.

27. OPS, WFP. United Nations World Food Programme, OAHO. Nutritional state of food and health conditions of the internally displaced population by the violence in six subregions of the country. Final report. Bogota, Colombia. December 2005. Available in http://documents.wfp.org/stellent/ groups/public/documents/liaison_offices/wfp086486.pdf. Retrieved March 2015.

28. Morrissey TW. Trajectories of growth in body mass index across childhood: Associations with maternal and paternal employment. Soc Sci Med. 2013;95:60-8.

29. Ruel MT, Levin CE, Armar-Klemesu M, Maxwell D, Morris SS. Good care practices can mitigate the negative effects of poverty and low maternal schooling on children's nutritional status:evidence from Accra. World Dev. 1999; 27(11): 1993-2009.

30. Vázquez L, Sánchez LA. Doctor Without Borders. Nutritional diagnosis of the altos de Cauza 2001. Municipality of Soacha, Cundinamarca (Colombia). Available in: http://www. disaster-info.net/desplazados/informes/msf/diagnosticonutricional.htm. Retrieved May 2015.

31. Herrán Falla OF, Prada Gómez GE, Patiño Benavides GA. Why are under-12-year-old infants malnourished in $\mathrm{Bu}$ caramanga?. Health department UIS 2001; 33(1):50-6.

32. Londoño A, Shirley S. Malnutrition risk indicators associated with a set of knowlegde and food procedures in preschool children from the lower social class in Calarcá. 2006-2007. Rev Gerenc Polit Salud 2009; 8(15):77-90.

33. Alcaraz G, Bernal C, Cornejo W, Figueroa $N$, Múnera $M$. Nutritional state and the living conditions of under-fiveyear-old children from the urban area of Turbo, Antioquia, Colombia, 2004. Biomedical 2008; 28(1): 87-96. 